\title{
Glucuretic effects and renal safety of dapagliflozin in patients with type 2 diabetes
}

\author{
Deborah Hinnen
}

Abstract: Dapagliflozin is a selective sodium-glucose cotransporter 2 (SGLT2) inhibitor approved as a treatment for type 2 diabetes mellitus (T2DM) in the United States, the European Union and other countries. Dapagliflozin increases renal glucose excretion in an insulin-independent manner, and its mechanism of action is complementary to those of other antidiabetes medications. When used as monotherapy or in combination with other oral antidiabetes medications or insulin, dapagliflozin improves glycemic measures in patients with T2DM. Dapagliflozin treatment is also associated with weight reduction and a decrease in blood pressure, both of which may be beneficial in patients with T2DM. Because of its mechanism of action, dapagliflozin has a low intrinsic propensity to cause hypoglycemia. Overall, dapagliflozin is well tolerated, with the frequency of most adverse events similar to that seen with placebo. Cases of genital infections and, in some studies, urinary tract infections have been more frequent in dapagliflozin-treated groups compared with placebo groups. In the clinical development program, more cases of newly diagnosed bladder cancer were reported for patients treated with dapagliflozin (0.17\%) compared with placebo or comparator $(0.03 \%)$. Although there were not enough cases to determine causality, dapagliflozin should not be used in patients with bladder cancer and should be used with caution in patients with a history of bladder cancer. Dapagliflozin may decrease glomerular filtration rate (GFR), especially in elderly patients and patients with impaired renal function. Renal function should be monitored before initiation of dapagliflozin. Dapagliflozin should not be used in patients with an estimated GFR $<60 \mathrm{ml} / \mathrm{min} / 1.73 \mathrm{~m}^{2}$. No cardiovascular safety signals have been detected for dapagliflozin, and a long-term cardiovascular outcomes study is ongoing. Evidence from clinical trials suggests that dapagliflozin is a promising new treatment option for T2DM.

Keywords: dapagliflozin, diabetes, glucose, kidney, SGLT2 inhibitors

\section{Introduction}

Diabetes is a complex disease that affects 382 million adults worldwide [International Diabetes Federation, 2013] and 29 million in the United States [Centers for Disease Control and Prevention, 2014]. Type 2 diabetes mellitus (T2DM) accounts for $>90 \%$ of newly diagnosed cases of diabetes and is a major cause of heart disease, stroke and kidney failure [Centers for Disease Control and Prevention, 2011]. Although there are numerous classes of antidiabetes drugs, $47 \%$ of US adults with diabetes do not attain a glycated hemoglobin (HbA1c) of $<7 \%$ [Stark Casagrande et al. 2013], a goal proposed by some national and international medical societies for many patients with T2DM [American Diabetes Association, 2014]. Owing to the progressive nature of T2DM [Fonseca, 2009], most patients will eventually require pharmacotherapy in addition to diet and exercise modification to achieve and maintain personal glycemic goals [Inzucchi et al. 2012]. Pharmacotherapy should be individualized based on efficacy, cost, side effects, comorbidities, potential for hypoglycemia and patient preference. Therapy will likely need to be intensified with additional complementary acting antidiabetes agents during the course of the disease to achieve and maintain glycemic control [American Diabetes Association, 2014; Garber et al. 2013].
Ther Adv Endocrinol Metab

2015, Vol. 6(3) 92-102

DOI: $10.1177 /$ 2042018815575273

(c) The Author(s), 2015. Reprints and permissions: http://www.sagepub.co.uk/ journalsPermissions.nav

Correspondence to: Deborah Hinnen, APN, BC-ADM, CDE, FAAN, FAADE

Memorial Hospital Diabetes Center, University of Colorado Health, 175 South Union Boulevard, Suite 305, Colorado Springs, CO 80190, USA Deborah.Hinnena uchealth.org 
In recent years, several new treatments have been developed that provide additional options for the individualized treatment of patients with T2DM [Garber et al. 2013]. Sodium-glucose cotransporter 2 (SGLT2) inhibitors, exemplified by three recently approved drugs - canagliflozin, dapagliflozin and empagliflozin - are the newest class of antidiabetes drugs [Bays, 2013]. Unlike other major antidiabetes drug classes, SGLT2 inhibitors act independently of insulin secretion or action [List and Whaley, 2011] and are therefore complementary to existing insulin-dependent therapies.
The purpose of this review is to discuss the mechanism of action of SGLT2 inhibitors and the efficacy of dapagliflozin in patients with T2DM. Because the mechanism of action of SGLT2 inhibitors is to inhibit glucose reabsorption by the kidney, the renal safety of dapagliflozin is discussed here as well.

\section{Mechanism of action of SGLT2 inhibitors}

SGLT2 is a sodium-dependent glucose transporter that is located in the proximal tubule of the kidney (Figure 1) and is responsible for the reabsorption of

\section{Normal physiology of renal glucose reabsorption}

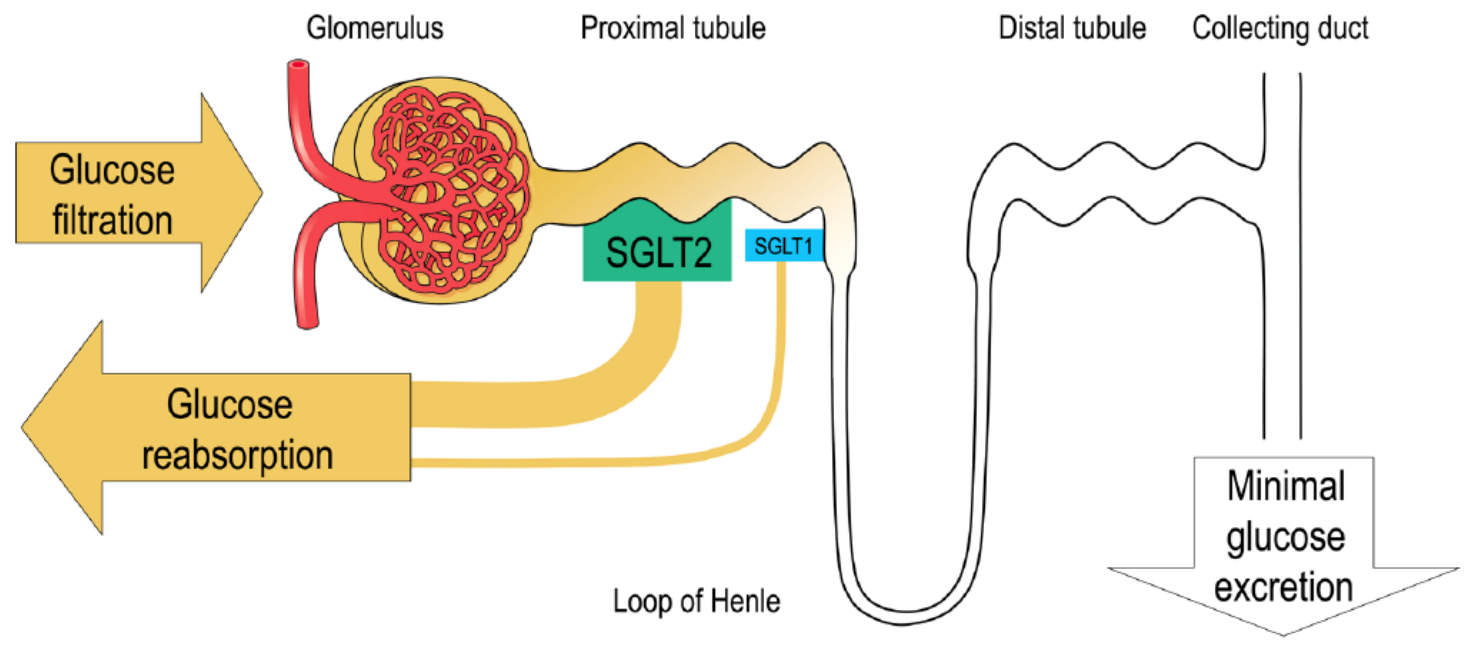

\section{SGLT2 inhibitors reduce renal glucose reabsorption}

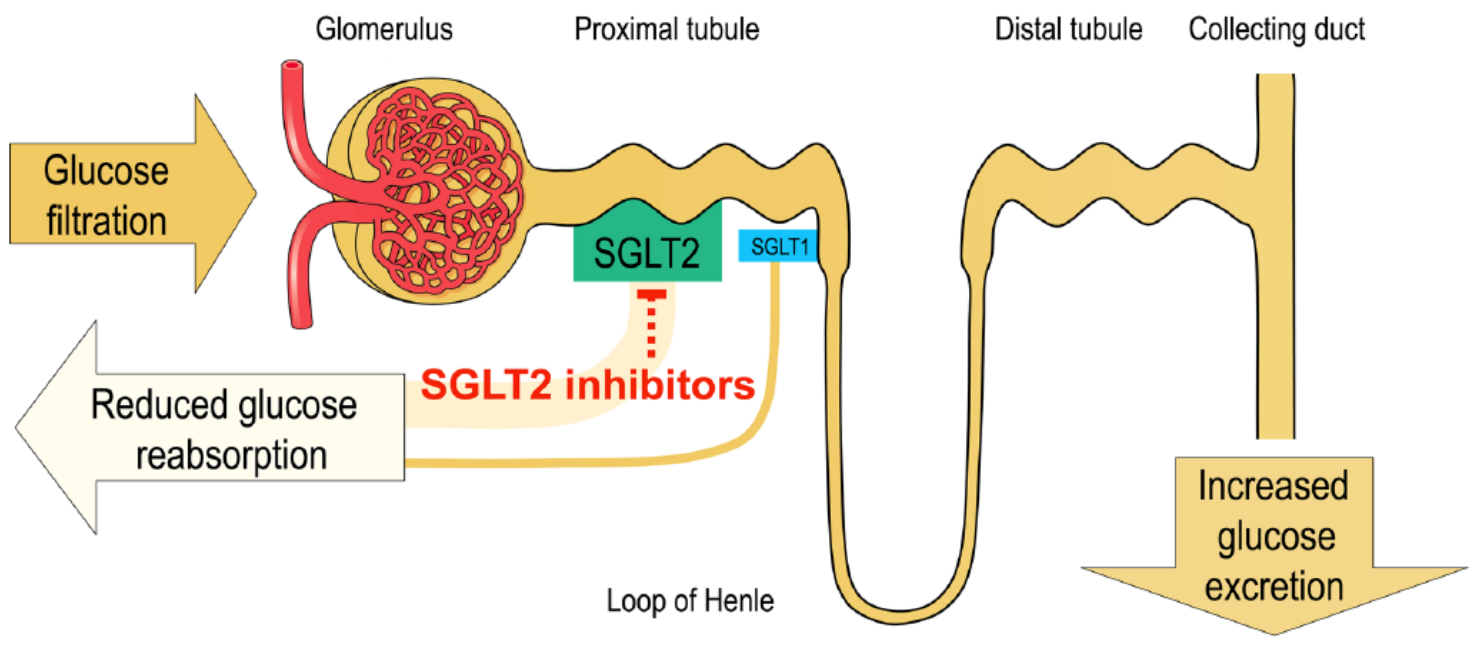

Figure 1. Mechanism of action of SGLT2 inhibitors.

SGLT1, sodium-glucose cotransporter 1; SGLT2, sodium-glucose cotransporter 2.

Reproduced with permission from Freeman [2013]. 
the majority $(\sim 90 \%)$ of the glucose that is filtered by the kidneys [Bakris et al. 2009]. The kidneys play an important role in glucose homeostasis. At normal plasma glucose concentrations, the kidneys reabsorb virtually all of the glucose that is filtered, such that urinary excretion of glucose is near zero [Bakris et al. 2009]. As plasma glucose concentration rises above $\sim 180 \mathrm{mg} / \mathrm{dl}$, more glucose is filtered, the reabsorptive capacity of the kidneys is exceeded, and glucose that is not reabsorbed is excreted in the urine [Defronzo et al. 2013]. Interestingly, in individuals with T2DM, the kidneys' capacity to reabsorb glucose is increased [Defronzo et al. 2013], which further contributes to the existing hyperglycemia. Thus, inhibition of SGLT2 represents a novel mechanism to reduce hyperglycemia by increasing renal glucose excretion. Because renal glucose excretion depends on both glomerular filtration rate (GFR) and plasma glucose concentrations, the ability of SGLT2 inhibitors to increase glucose excretion in the urine, and thus decrease plasma glucose concentrations, is attenuated in patients with reduced GFR [Barnett et al. 2014; Kohan et al. 2014; Yale et al. 2013].

The mechanism of action of SGLT2 inhibitors does not depend on insulin secretion or action [List and Whaley, 2011], which offers a number of potential benefits. First, there is less risk of hypoglycemic events with this drug class compared with, for example, the sulfonylureas (SUs), which are insulin secretagogues [Vasilakou et al. 2013]. Second, because they do not depend on $\beta$-cell function or tissue insulin sensitivity, SGLT2 inhibitors are effective throughout the progression of T2DM, from patients newly diagnosed with T2DM to those with long-standing disease, as long as there is adequate renal function [Zhang et al. 2010]. Additional benefits of SGLT2 inhibitors include body weight reduction [Vasilakou et al. 2013], associated with the loss of calories through increased renal excretion of glucose [Bolinder et al. 2012] and blood pressure reduction, possibly the result, at least in part, of a combination of weight loss and mild osmotic diuresis [Lambers Heerspink et al. 2013]. However, the pharmacologically induced excretion of urine with high concentrations of glucose may increase the risk of genital and urinary tract infections (UTIs) in patients with T2DM [Vasilakou et al. 2013].

\section{Dapagliflozin efficacy in patients with T2DM}

Dapagliflozin was approved in the EU in 2012 and in the US in 2014 as an adjunct to diet and exercise to improve glycemic control in adults with T2DM. In the US, the recommended starting dose is $5 \mathrm{mg}$ /day, which can be increased to 10 $\mathrm{mg} /$ day in patients who require additional glycemic control [Bristol-Myers Squibb and AstraZeneca, 2014]. In the EU, the recommended dose is $10 \mathrm{mg} /$ day. Dapagliflozin should not be used in patients with an estimated GFR (eGFR) $<60 \mathrm{ml} / \mathrm{min} / 1.73 \mathrm{~m}^{2}$ [Bristol-Myers Squibb and AstraZeneca, 2014]. No important drug interactions have been observed with other oral antidiabetes drugs (OADs) such as metformin, pioglitazone, sitagliptin and glimepiride [Kasichayanula et al. 2011], or with the cardiovascular (CV) drugs valsartan, simvastatin, warfarin and digoxin, when co-administered with dapagliflozin [Kasichayanula et al. 2012]. No adjustment in dapagliflozin dose is required in patients with mild renal impairment (eGFR $60-90 \mathrm{ml} / \mathrm{min} / 1.73 \mathrm{~m}^{2}$ ) [Bristol-Myers Squibb and AstraZeneca, 2014].

In the clinical development program, the effects of dapagliflozin on glycemic parameters when used as monotherapy in treatment-naïve patients [Ferrannini et al. 2010] and as add-on therapy to other antidiabetes drugs, including metformin [Bailey et al. 2010], SUs [Strojek et al. 2011], thiazolidinediones (TZDs) [Rosenstock et al. 2012], dipeptidyl peptidase-4 (DPP-4) inhibitors [Jabbour et al. 2014] and insulin [Wilding et al. 2012], were measured. Dapagliflozin was compared with the SU glipizide as add-on therapy to metformin [Nauck et al. 2011]. Dapagliflozin was also compared as an initial combination therapy with metformin extended release (XR) versus dapagliflozin or metformin XR alone [Henry et al. 2012]. A summary of these clinical trials is presented in Table 1.

In treatment-naïve patients with inadequate glycemic control with diet and exercise modification, monotherapy with dapagliflozin 5 and $10 \mathrm{mg} /$ day significantly reduced $\mathrm{HbA} 1 \mathrm{c}$ (placebo corrected) by $0.54 \%$ and $0.66 \%$, respectively, after 24 weeks [Ferrannini et al. 2010]. Fasting plasma glucose (FPG) was reduced by 20 and $25 \mathrm{mg} / \mathrm{dl}$ by dapagliflozin 5 and $10 \mathrm{mg} /$ day, respectively, compared with placebo. In other studies, initial therapy with dapagliflozin 5 or $10 \mathrm{mg} /$ day alone, metformin $\mathrm{XR}$ alone, or the combination of dapagliflozin and metformin XR was assessed in treatmentnaïve patients. The combination of dapagliflozin and metformin XR significantly reduced $\mathrm{HbAlc}$ by $0.70 \%$ with dapagliflozin $5 \mathrm{mg} /$ day + MET and $0.54 \%$ with dapagliflozin $10 \mathrm{mg} /$ day + MET 


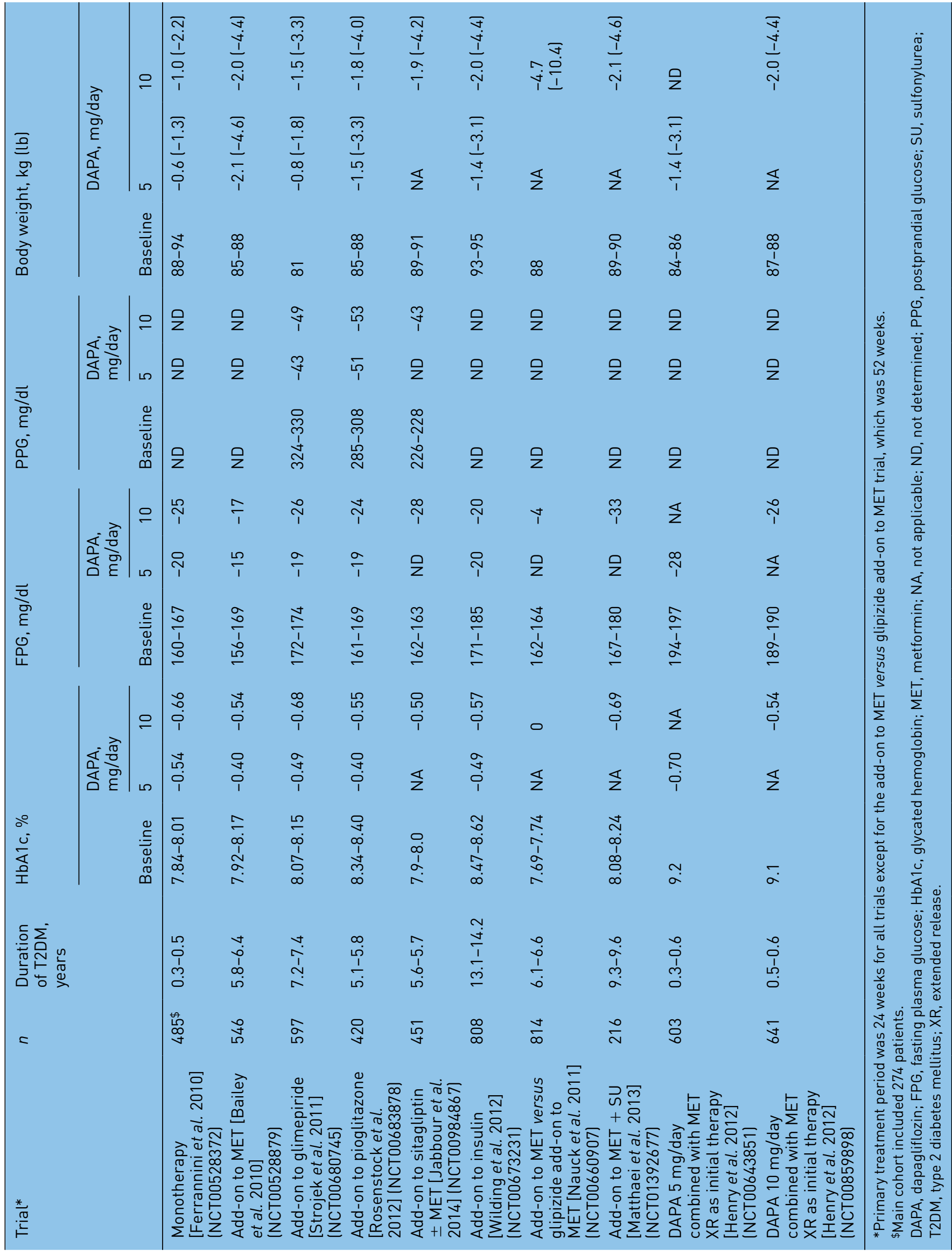


and FPG by 27 and $26 \mathrm{mg} / \mathrm{dl}$, respectively, versus metformin XR alone. Dapagliflozin $10 \mathrm{mg} /$ day alone was noninferior to metformin $\mathrm{XR}$ alone in reducing HbA1c [Henry et al. 2012]. Body weight was significantly reduced with dapagliflozin 5 or $10 \mathrm{mg} /$ day plus metformin XR by 1.4 and $2.0 \mathrm{~kg}$ (3.1 and $4.4 \mathrm{lb}$ ), respectively, compared with metformin XR alone. In patients with more advanced disease (T2DM duration of 5.1-14.2 years), dapagliflozin, when used as add-on therapy to other OADs or insulin [Bailey et al. 2010; Jabbour et al. 2014; Rosenstock et al. 2012; Strojek et al. 2011; Wilding et al. 2012], reduced HbA1c compared with placebo by $0.40 \%$ to $0.69 \%$, FPG by 15 to $33 \mathrm{mg} / \mathrm{dl}$, and body weight by up to approximately $2.0 \mathrm{~kg}(4.4 \mathrm{lb})$ (Table 1). Dapagliflozin also reduced 2-hour postprandial glucose (PPG) compared with placebo by -43 to $-53 \mathrm{mg} / \mathrm{dl}$ when used as add-on to glimepiride [Strojek et al. 2011], add-on to pioglitazone [Rosenstock et al. 2012] or add-on to sitagliptin with or without metformin [Jabbour et al. 2014]. Long-term extensions of the add-on to metformin [Bailey et al. 2013] and add-on to insulin studies [Wilding et al. 2014] showed that the improvements in glycemic parameters and reduction in body weight were maintained for up to 2 years.

In a study designed to compare the efficacy of dapagliflozin at a maximum dose of $10 \mathrm{mg} /$ day with glipizide at a maximum dose of up to $20 \mathrm{mg}$ / day in patients inadequately controlled with metformin [Nauck et al. 2011], the reduction in $\mathrm{HbA} 1 \mathrm{c}$ after 52 weeks of treatment was identical $(0.52 \%)$ in both treatment groups, and there was no difference in the decrease in FPG between the groups. Notably, body weight increased with glipizide by $1.4 \mathrm{~kg}(3.1 \mathrm{lb})$ and decreased by $3.2 \mathrm{~kg}$ $(7.1 \mathrm{lb})$ with dapagliflozin, resulting in a difference of $4.7 \mathrm{~kg}(10.4 \mathrm{lb})$ between treatment groups.

National and international diabetes societies recommend that addition of a third noninsulin agent be considered as a treatment option in some patients when dual therapy fails to achieve or maintain glycemic control [Garber et al. 2013; International Diabetes Federation, 2012; Inzucchi et al. 2012]. The efficacy of dapagliflozin $10 \mathrm{mg} /$ day versus placebo as add-on therapy in patients poorly controlled on metformin ( $\geqslant 1500 \mathrm{mg} /$ day) and a maximum tolerated dose of an SU was assessed [Matthaei et al. 2013 ]. Compared with placebo, after 24 weeks, dapagliflozin significantly reduced HbAlc (0.69\%), FPG (33 mg/dl) and body weight $[2.1 \mathrm{~kg}(4.6 \mathrm{lb})]$. Another study compared the dual addition of dapagliflozin 10 $\mathrm{mg} /$ day and the DPP-4 inhibitor saxagliptin $5 \mathrm{mg} /$ day versus the addition of dapagliflozin $10 \mathrm{mg} /$ day and placebo or saxagliptin $5 \mathrm{mg} /$ day and placebo to metformin XR in patients inadequately controlled with metformin XR alone (Rosenstock et al. 2015). After 24 weeks, the adjusted mean change from baseline in HbAlc was significantly greater with saxagliptin and dapagliflozin add-on $(-1.47 \%)$ than with saxagliptin add-on alone $(-0.88 \%, p<0.0001)$ or dapagliflozin add-on alone $(-1.20 \%, p<0.02)$. Changes in FPG were $-38 \mathrm{mg} / \mathrm{dl}$ with saxagliptin and dapagliflozin addon, $-14 \mathrm{mg} / \mathrm{dl}$ with saxagliptin add-on and -32 $\mathrm{mg} / \mathrm{dl}$ with dapagliflozin add-on. There was no change in body weight in the saxagliptin add-on group, whereas body weight was reduced by 2.1 $\mathrm{kg}(4.6 \mathrm{lb})$ in the saxagliptin and dapagliflozin group and $2.4 \mathrm{~kg}(5.3 \mathrm{lb})$ in the dapagliflozin group.

In patients with $\mathrm{T} 2 \mathrm{DM}$ and moderate renal impairment $(89-94 \%$ of patients in the 3 study arms had an eGFR $\geqslant 30$ and $<60 \mathrm{ml} / \mathrm{min} / 1.73$ $\mathrm{m}^{2}$ ) who continued on their existing antidiabetes medications, the change in $\mathrm{HbA} 1 \mathrm{c}$ after 24 weeks with dapagliflozin $5 \mathrm{mg} /$ day $(-0.41 \%)$ and $10 \mathrm{mg} /$ day $(-0.44 \%)$ was not statistically different from that with placebo $(-0.32 \%)$ [Kohan et al. 2014]. Body weight was reduced by 1.3 to $1.7 \mathrm{~kg}$ (2.9 to $3.7 \mathrm{lb}$ ) in patients treated with dapagliflozin and was increased by $0.7 \mathrm{~kg}(1.5 \mathrm{lb})$ in the placebo group. A post hoc analysis found larger adjusted mean (SE) change from baseline compared with placebo in $\mathrm{HbA} 1 \mathrm{c}$ [ $-0.37 \%(0.23 \%)$ and $-0.33 \%$ $(0.24 \%)]$ for dapagliflozin 5 and $10 \mathrm{mg} /$ day, respectively) and FPG [-24.8 (12.4) and -24.4 (12.7) $\mathrm{mg} / \mathrm{dl}]$ in patients with eGFR of $\geqslant 45$ and $<60 \mathrm{ml} / \mathrm{min} / 1.73 \mathrm{~m}^{2}$ than for those with eGFR $\geqslant 30$ and $<45 \mathrm{ml} / \mathrm{min} / 1.73 \mathrm{~m}^{2}$ [HbAlc, $0.05 \%$ $(0.21 \%)$ and $0.07 \%(0.21 \%)$; FPG, $-2.7(12.1)$ and $-1.5(11.8) \mathrm{mg} / \mathrm{dll}])$. The observed decrease in dapagliflozin efficacy with worsening renal function is characteristic of SGLT2 inhibitors [Barnett et al. 2014; Yale et al. 2013] owing to their mechanism of action.

As noted above, moderate weight reduction has been a consistent finding in patients treated with dapagliflozin. Because dapagliflozin appears to have a mild diuretic effect [Lambers Heerspink et al. 2013], the weight loss could result from fluid loss secondary to osmotic diuresis; it could also be attributed to caloric loss resulting from increased glucose excretion or to both. A study 
was performed to determine whether body weight reduction with dapagliflozin was the result of changes in body composition [Bolinder et al. 2012]. Patients with T2DM were treated with dapagliflozin $10 \mathrm{mg} /$ day or placebo in addition to open-label metformin. Change in fat mass was assessed by dual-energy X-ray absorptiometry. At the end of 24 weeks, placebo-corrected change in body weight with dapagliflozin was $-2.1 \mathrm{~kg}(-4.6$ $\mathrm{lb} ; p<0.0001)$. A reduction in fat mass accounted for approximately two-thirds of the total weight loss. The changes in body weight and fat mass with dapagliflozin were sustained for a total of 102 weeks of treatment, at which time placebocorrected weight loss was $2.4 \mathrm{~kg}(5.3 \mathrm{lb})$ and reduction in fat mass accounted for $68 \%$ of the reduction in body weight [Bolinder et al. 2014]. These results suggest that, although the initial reduction in body weight may be due, in large part, to osmotic diuresis, the gradual continuous reduction and subsequent stabilization of body weight is likely the result of caloric loss via increased glucose excretion [Lambers Heerspink et al. 2013].

In most studies with dapagliflozin, modest reductions in blood pressure have been reported, probably the result of a mild diuretic-like effect of dapagliflozin [Lambers Heerspink et al. 2013]. In patients with T2DM and hypertension being treated with OADs and an angiotensin-converting enzyme inhibitor (ACEi) or angiotensin receptor blocker (ARB), dapagliflozin $10 \mathrm{mg}$ /day significantly reduced seated systolic blood pressure versus placebo by $3.1 \mathrm{~mm} \mathrm{Hg}$ after 12 weeks of treatment [Weber et al. 2013a]. In a similarly designed 12-week study in patients with hypertension and T2DM receiving OADs, an ACEi or $\mathrm{ARB}$, and a second antihypertensive agent, dapagliflozin $10 \mathrm{mg} /$ day significantly reduced seated systolic blood pressure versus placebo by $4.3 \mathrm{~mm}$ Hg [Weber et al. 2013b]. In both studies, asymptomatic orthostatic hypotension occurred in $<3.5 \%$ of patients.

\section{Overall safety}

In the studies listed in Table 1, the proportion of patients reporting $\geqslant 1$ adverse event (AE) was similar to or slightly higher in patients treated with dapagliflozin compared with those receiving placebo or a comparator drug [Bailey et al. 2010; Ferrannini et al. 2010; Henry et al. 2012; Jabbour et al. 2014; Matthaei et al. 2013; Nauck et al. 2011; Rosenstock et al. 2012; Strojek et al. 2011;
Wilding et al. 2012]. The most common AEs included nasopharyngitis, diarrhea, headache and upper respiratory tract infections.

Hyperglycemia and diabetes are associated with an increased risk of genital and urinary tract infections [Benfield et al. 2007; De Leon et al. 2002]. Because glucosuria may play a role in the development of genital and urinary tract infections [Geerlings et al. 2014], patients were actively monitored throughout dapagliflozin clinical trials for signs and symptoms suggestive of UTI and genital infections [Bailey et al. 2010]. In data pooled from 12 double-blind clinical trials of dapagliflozin, confirmed cases of genital infections were more frequent in patients receiving dapagliflozin $5 \mathrm{mg} /$ day (6\%) and $10 \mathrm{mg} /$ day (5\%) than in those receiving placebo or comparator (1\%) [Johnsson et al. 2013a]. In the dapagliflozin 5 and $10 \mathrm{mg} /$ day groups, genital infections were more common in women $(8 \%$ and $7 \%$, respectively) than in men (3\% and 3\%). For all groups, diagnosis of genital infections was more prevalent in patients with a history of recurrent genital infections. Confirmed cases of UTI in the 12 -study pool were somewhat more frequent in the dapagliflozin $5 \mathrm{mg} /$ day group (6\%), but similar in the dapagliflozin $10 \mathrm{mg} /$ day group (4\%) to the placebo or comparator groups (4\%) [Johnsson et al. 2013b]. The increase in genital infections and, in some studies, UTIs has been reported for other SGLT2 inhibitors [Häring et al. 2013; Nicolle et al. 2014; Nyirjesy et al. 2012] and appears to be a class effect. Patients, especially those with a history of genital infections, should be informed of the increased risk of genital mycotic infections and should be monitored and treated accordingly [Bristol-Myers Squibb and AstraZeneca, 2014].

The incidence of hypoglycemia with dapagliflozin was low and similar to that with placebo in most studies. Hypoglycemic events were reported more frequently with dapagliflozin 5 and $10 \mathrm{mg} /$ day than with placebo when dapagliflozin was added on to insulin (56\% and 54\% versus $52 \%$, respectively) [Wilding et al. 2012] or glimepiride ( $7 \%$ and $8 \%$ versus $5 \%$ ) [Strojek et al. 2011] or with dapagliflozin $10 \mathrm{mg} /$ day added on to metformin plus an SU (13\% versus 4\%) [Matthaei et al. 2013]. Of note, hypoglycemia was approximately 10-fold less frequent with dapagliflozin $(3.4 \%)$ compared with glipizide $(39.7 \%)$ as add-on therapy to metformin [Nauck et al. 2011]. 
Major hypoglycemic episodes, defined as symptomatic episodes in which the patient required external assistance owing to severe impairment in consciousness or behavior, had plasma glucose concentration $<54 \mathrm{mg} / \mathrm{dl}$, and promptly recovered after receiving glucose or glucagon, were rare with dapagliflozin. One major hypoglycemic episode occurred with dapagliflozin $10 \mathrm{mg} /$ day versus none with placebo during the 48 weeks of treatment in the add-on to sitagliptin study [Jabbour et al. 2014]. In the add-on to insulin study, there were $2(0.9 \%)$ major episodes with dapagliflozin $5 \mathrm{mg} /$ day plus insulin, $3(1.5 \%)$ with dapagliflozin $10 \mathrm{mg} /$ day plus insulin, and $2(1 \%)$ with placebo plus insulin.

In 13 phase IIb and III placebo-controlled trials, events of volume depletion (e.g. orthostatic hypotension, syncope, dehydration) were infrequent but slightly more common with dapagliflozin 10 $\mathrm{mg} /$ day $(1.9 \%)$ than with placebo $(1.4 \%)$ [Food and Drug Administration, 2013]. Events were more frequent with either dapagliflozin or placebo in patients $\geqslant 65$ years old $(2.3 \%$ and $1.7 \%$, respectively), those receiving loop diuretics (3\% and $2.7 \%$ ), and those with moderate renal impairment $(3.2 \%$ and $2.4 \%)$. Volume status should be assessed and corrected in elderly patients, patients with renal impairment or low systolic blood pressure, and patients taking diuretics before initiating dapagliflozin therapy [Bristol-Myers Squibb and AstraZeneca, 2014].

In this same placebo-controlled trial pool, the proportion of patients with AEs leading to discontinuation was slightly higher with dapagliflozin $(8.5 \%)$ than with placebo $(7.4 \%)$ [Food and Drug Administration, 2013]. AEs leading to discontinuation that were more frequent in dapagliflozin-treated patients than in those receiving placebo included changes in renal function and genital infections or UTIs.

In data from the dapagliflozin clinical development program presented to the US Food and Drug Administration (FDA) in 2013, an imbalance in bladder cancer was observed with dapagliflozin. In 22 clinical studies, newly diagnosed cases of bladder cancer were reported in $1 / 3512$ patients $(0.03 \%)$ receiving placebo or comparator and in $10 / 6045$ patients $(0.17 \%)$ treated with dapagliflozin [Food and Drug Administration, 2013]. There were 6 cases of bladder cancer with dapagliflozin and 1 case with placebo or comparator in patients who were exposed to study drug for $<1$ year at the time of diagnosis of bladder cancer; the remaining 4 cases occurred within 1-2 years of initiation of dapagliflozin treatment. There were too few cases and insufficient data to determine causality [Food and Drug Administration, 2013]. Dapagliflozin should not be used in patients with active bladder cancer and should be used with caution in those with a history of bladder cancer, taking into consideration the benefits of glycemic control versus unknown risks for cancer recurrence [Bristol-Myers Squibb and AstraZeneca, 2014].

A meta-analysis of 21 phase IIb/III trials was performed to assess the CV safety of dapagliflozin as per the 2008 FDA guidance on CV safety in antidiabetes drug development [Food and Drug Administration, 2008]. The prespecified primary endpoint was a composite of the time to first event of $\mathrm{CV}$ death, myocardial infarction, stroke, or hospitalization for unstable angina. As per the guidance criteria, there was no unacceptable increase in CV risk with dapagliflozin compared with placebo or comparator [Food and Drug Administration, 2013]. A secondary analysis of CV major AEs (MACE) (a composite of CV death, nonfatal myocardial infarction, or nonfatal stroke) also showed no increased risk with dapagliflozin. A long-term CV outcomes trial to assess the effect of dapagliflozin on the incidence of CV events in patients with T2DM and at high risk for $\mathrm{CV}$ events is ongoing [ClinicalTrials.gov identifier: NCT01730534].

\section{Renal safety}

Because the mechanism of action of SGLT2 inhibitors is to inhibit glucose reabsorption in the kidney, renal safety is of special interest. In an exploratory study in patients with T2DM and eGFR $>60 \mathrm{ml} / \mathrm{min} / 1.73 \mathrm{~m}^{2}$, dapagliflozin $10 \mathrm{mg} /$ day for 12 weeks reduced GFR as measured by the plasma disappearance of iohexol from baseline by $10.8 \%$ compared with $2.9 \%$ with placebo [Lambers Heerspink et al. 2013]. Mean eGFR decreased from baseline up to week 2 and, unlike measured GFR, returned to values close to baseline by 12 weeks (mean change -0.5 versus $2.0 \mathrm{ml} /$ $\mathrm{min} / 1.73 \mathrm{~m}^{2}$ with dapagliflozin versus placebo). In an analysis of 12 placebo-controlled randomized trials involving $>4000$ patients, most of whom had either normal renal function $(37-40 \%$ of patients, eGFR $>90 \mathrm{ml} / \mathrm{min} / 1.73 \mathrm{~m}^{2}$ ) or mild renal impairment $(53-54 \%$ of patients; eGFR 60-90 $\mathrm{ml} / \mathrm{min} / 1.73 \mathrm{~m}^{2}$ ), dapagliflozin was 
associated with a small reduction in eGFR at week 1 that returned to baseline by week 24 and was maintained at baseline values for up to 2 years [Ptaszynska et al. 2014]. It has been postulated that the acute reductions in eGFR may reflect reversible hemodynamic effects, such as a decrease in plasma volume or blood pressure, or autoregulation through tubuloglomerular feedback due to increased delivery of sodium to the macula densa rather than permanent changes in renal function [Lambers Heerspink et al. 2013]. Acute reductions in eGFR followed by stabilization have been shown with other SGLT2 inhibitors [Cefalu et al. 2013] and appear to be a class effect.

In the aforementioned 12 clinical trials, there were no changes from baseline in serum sodium, potassium, bicarbonate, chloride or calcium with dapagliflozin or placebo. Small increases in serum magnesium and phosphate were reported. There were no changes in albumin excretion (expressed as the urinary albumin/creatinine ratio) [Ptaszynska et al. 2014], a marker of kidney functional damage [Heerspink et al. 2011].

In a study in patients with T2DM and moderate renal impairment (eGFR 30-60 ml/min/1.73 m²), dapagliflozin 5 and $10 \mathrm{mg} /$ day caused a decline in eGFR over the first 1-2 weeks, followed by stabilization or small increases in eGFR through 104 weeks of therapy (albeit with no return to baseline values), whereas in the placebo group, eGFR appeared to decline over this period [Kohan et al. 2014]. The change from baseline in eGFR at 104 weeks was similar across groups (placebo, -2.4 $\mathrm{ml} / \mathrm{min} / 1.73 \mathrm{~m}^{2}$; dapagliflozin $5 \mathrm{mg} /$ day, $-1.7 \mathrm{ml} /$ $\mathrm{min} / 1.73 \mathrm{~m}^{2}$; dapagliflozin $10 \mathrm{mg} /$ day, $-3.5 \mathrm{ml} /$ $\min / 1.73 \mathrm{~m}^{2}$ ). No changes from baseline were observed for mean serum sodium, potassium, bicarbonate, chloride or calcium. There was a higher incidence of hyperphosphatemia in patients treated with dapagliflozin $5 \mathrm{mg} /$ day $(16 \%)$ and $10 \mathrm{mg} /$ day $(14 \%)$ compared with those receiving placebo ( $8 \%$ ). Urinary albumin/ creatinine ratio increased from baseline with dapagliflozin $5 \mathrm{mg} /$ day $(78 \mathrm{mg} / \mathrm{g})$ and placebo $(70$ $\mathrm{mg} / \mathrm{g}$ ) and decreased with dapagliflozin $10 \mathrm{mg} /$ day $(-12 \mathrm{mg} / \mathrm{g})$.

In 13 phase IIb and III placebo-controlled trials with durations from 12 to 104 weeks $(n=3982)$, AEs related to renal function (e.g. decreased creatinine clearance, increased blood creatinine, decreased urine flow, anuria) were more common in patients treated with dapagliflozin $10 \mathrm{mg} /$ day (7\%) than in those receiving placebo (4\%) [Food and Drug Administration, 2013]. Patients $\geqslant 65$ years old were at an increased risk for AEs related to renal function (dapagliflozin $10 \mathrm{mg} / \mathrm{day}, 14 \%$; placebo, $8 \%$ ), as were those with moderate renal impairment (eGFR 30 to $<60 \mathrm{ml} / \mathrm{min} / 1.73 \mathrm{~m}^{2}$; dapagliflozin $10 \mathrm{mg} /$ day, $28 \%$; placebo, $16 \%$ ). Renal function should be assessed before initiating dapagliflozin. Dapagliflozin should not be used in patients with an eGFR $<60 \mathrm{ml} / \mathrm{min} / 1.73 \mathrm{~m}^{2}$ [Bristol-Myers Squibb and AstraZeneca, 2014].

Across all phase IIb and III clinical trials ( $n=$ 9339), serious AEs (SAEs) of renal impairment occurred in $9(0.2 \%)$ dapagliflozin-treated patients and in $5(0.1 \%)$ patients receiving placebo or control [Food and Drug Administration, 2013].

\section{Summary}

Dapagliflozin is a member of a new class of antidiabetes agents, the SGLT2 inhibitors, that reduce plasma glucose concentrations by inhibiting renal glucose reabsorption. Because the efficacy of dapagliflozin is not affected by either $\beta$-cell dysfunction or insulin resistance, it improves glycemic control in a wide range of patients, from treatment-naïve patients to those with long-standing disease, as long as renal function is adequate. Moreover, dapagliflozin can be used with other OADs and with insulin to gain additional efficacy.

Dapagliflozin is associated with weight reduction that is maintained for at least 2 years, which may be a benefit for overweight and obese patients with $\mathrm{T} 2 \mathrm{DM}$ as well as a means to mitigate the weight gain usually seen with SU and insulin therapy. Reduction in blood pressure with dapagliflozin is an additional potential benefit in view of the high prevalence of hypertension in patients with T2DM.

In clinical trials, dapagliflozin was generally well tolerated, with genital infections and UTIs being the most common AEs. Patients should be informed of the signs and symptoms of these infections and the need to seek treatment should they occur. Dapagliflozin has a low intrinsic propensity to cause hypoglycemia. However, hypoglycemic events were more frequent when dapagliflozin was added to insulin or SU therapy.

In a meta-analysis of short-term clinical trials, there was no increased risk of adverse CV events with dapagliflozin treatment. Dapagliflozin can 
decrease eGFR, especially in elderly patients and in patients with impaired renal function. Renal function should be monitored before initiation of dapagliflozin. Volume-related AEs were infrequent with dapagliflozin. The volume status of elderly patients and those receiving diuretics or with renal impairment should be monitored. Dapagliflozin should not be used in patients with bladder cancer, and caution should be exercised in patients with a history of bladder cancer.

\section{Conclusion}

Dapagliflozin is an SGLT2 inhibitor that improves glycemic control in patients inadequately controlled by diet and exercise modification or antidiabetes medications. Additional benefits of dapagliflozin include reductions in body weight and blood pressure. Dapagliflozin poses a low risk of hypoglycemia, except when used with insulin or insulin secretagogues. The most common AEs in clinical trials were genital and urinary tract infections. No increase in CV risk was detected during the clinical development program, and an outcomes study is being conducted to assess the effects of dapagliflozin on CV events. Evidence from clinical trials suggests that dapagliflozin is a promising new treatment option for T2DM.

\section{Acknowledgements}

Editorial support was provided by Richard $M$. Edwards, $\mathrm{PhD}$, and Janet E. Matsuura, $\mathrm{PhD}$, from Complete Healthcare Communications, Inc., and was funded by Bristol-Myers Squibb and AstraZeneca.

\section{Conflict of interest statement}

D.H. is on the speakers bureau for AstraZeneca, Lilly, Boehringer Ingelheim and Janssen Pharmaceuticals.

\section{Funding}

Aside from funded editorial support, this research received no specific grant from any funding agency in the public, commercial, or not-forprofit sectors.

\section{References}

American Diabetes Association (2014) Standards of medical care in diabetes-2014. Diabetes Care 37: S14-80.

Bailey, C., Gross, J., Hennicken, D., Iqbal, N., Mansfield, T. and List, J. (2013) Dapagliflozin add-on to metformin in type 2 diabetes inadequately controlled with metformin: a randomized, doubleblind, placebo-controlled 102-week trial. BMC Med 11: 43 .

Bailey, C., Gross, J., Pieters, A., Bastien, A. and List, J. (2010) Effect of dapagliflozin in patients with type 2 diabetes who have inadequate glycaemic control with metformin: a randomised, double-blind, placebocontrolled trial. Lancet 375: 2223-2233.

Bakris, G., Fonseca, V., Sharma, K. and Wright, E. (2009) Renal sodium-glucose transport: role in diabetes mellitus and potential clinical implications. Kidney Int 75: 1272-1277.

Barnett, A., Mithal, A., Manassie, J., Jones, R., Rattunde, H., Woerle, H. et al. (2014) Efficacy and safety of empagliflozin added to existing antidiabetes treatment in patients with type 2 diabetes and chronic kidney disease: a randomised, double-blind, placebocontrolled trial. Lancet Diabetes Endocrinol 2: 369-384.

Bays, H. (2013) Sodium glucose co-transporter type 2 (SGLT2) inhibitors: targeting the kidney to improve glycemic control in diabetes mellitus. Diabetes Ther 4: 195-220.

Benfield, T., Jensen, J. and Nordestgaard, B. (2007) Influence of diabetes and hyperglycaemia on infectious disease hospitalisation and outcome. Diabetologia 50: 549-554.

Bolinder, J., Ljunggren, O., Johansson, L., Wilding, J., Langkilde, A., Sjostrom, C. et al. (2014) Dapagliflozin maintains glycaemic control while reducing weight and body fat mass over 2 years in patients with type 2 diabetes mellitus inadequately controlled on metformin. Diabetes Obes Metab 16: 159-169.

Bolinder, J., Ljunggren, O., Kullberg, J., Johansson, L., Wilding, J., Langkilde, A. et al. (2012) Effects of dapagliflozin on body weight, total fat mass, and regional adipose tissue distribution in patients with type 2 diabetes mellitus with inadequate glycemic control on metformin. $\mathcal{F}$ Clin Endocrinol Metab 97: 1020-1031.

Bristol-Myers Squibb and AstraZeneca (2014) Farxiga $^{\circledR}$ (dapagliflozin). Full prescribing information. Princeton, NJ, and Wilmington, DE: Bristol-Myers Squibb and AstraZeneca.

Cefalu, W., Leiter, L., Yoon, K., Arias, P., Niskanen, L., Xie, J. et al. (2013) Efficacy and safety of canagliflozin versus glimepiride in patients with type 2 diabetes inadequately controlled with metformin (CANTATA-SU): 52 week results from a randomised, double-blind, phase 3 non-inferiority trial. Lancet 382: 941-950.

Centers for Disease Control and Prevention (2011) National diabetes fact sheet: national estimates and general information on diabetes and prediabetes in the 
United States, 2011. Atlanta, GA: US Department of Health and Human Services, Centers for Disease Control and Prevention.

Centers for Disease Control and Prevention (2014) National diabetes statistics report: estimates of diabetes and its burden in the United States, 2014. Atlanta, GA: US Department of Health and Human Services, Centers for Disease Control and Prevention.

De Leon, E., Jacober, S., Sobel, J. and Foxman, B. (2002) Prevalence and risk factors for vaginal Candida colonization in women with type 1 and type 2 diabetes. BMC Infect Dis 2: 1 .

DeFronzo, R., Hompesch, M., Kasichayanula, S., Liu, X., Hong, Y., Pfister, M. et al. (2013) Characterization of renal glucose reabsorption in response to dapagliflozin in healthy subjects and subjects with type 2 diabetes. Diabetes Care 36: 3169-3176.

Ferrannini, E., Ramos, S., Salsali, A., Tang, W. and List, J. (2010) Dapagliflozin monotherapy in type 2 diabetic patients with inadequate glycemic control by diet and exercise: a randomized, double-blind, placebo-controlled, phase 3 trial. Diabetes Care 33: 2217-2224.

Fonseca, V. (2009) Defining and characterizing the progression of type 2 diabetes. Diabetes Care 32: S151-156.

Food and Drug Administration (2008) Guidance for industry: diabetes mellitus - evaluating cardiovascular risk in new antidiabetic therapies to treat type 2 diabetes. Silver Spring, MD: Center for Drug Evaluation and Research.

Food and Drug Administration (2013) FDA briefing document. NDA 202293. Dapagliflozin oral tablets, 5 and $10 \mathrm{mg}$. Advisory Committee Meeting, 12 December 2013.

Freeman, J. (2013) Review of insulin-dependent and insulin-independent agents for treating patients with type 2 diabetes mellitus and potential role for sodiumglucose co-transporter 2 inhibitors. Postgrad Med 125: 214-226.

Garber, A., Abrahamson, M., Barzilay, J., Blonde, L., Bloomgarden, Z., Bush, M. et al. (2013) American Association of Clinical Endocrinologists' comprehensive diabetes management algorithm 2013 consensus statement-executive summary. Endocr Pract 19: 536-557.

Geerlings, S., Fonseca, V., Castro-Diaz, D., List, J. and Parikh, S. (2014) Genital and urinary tract infections in diabetes: impact of pharmacologicallyinduced glucosuria. Diabetes Res Clin Pract 103: 373-381.

Häring, H., Merker, L., Seewaldt-Becker, E., Weimer, M., Meinicke, T., Woerle, H. et al.
(2013) Empagliflozin as add-on to metformin plus sulfonylurea in patients with type 2 diabetes: a 24-week, randomized, double-blind, placebocontrolled trial. Diabetes Care 36: 3396-3404.

Heerspink, H., Holtkamp, F., De Zeeuw, D. and Ravid, M. (2011) Monitoring kidney function and albuminuria in patients with diabetes. Diabetes Care 34(Suppl. 2): S325-S329.

Henry, R., Murray, A., Marmolejo, M., Hennicken, D., Ptaszynska, A. and List, J. (2012) Dapagliflozin, metformin XR, or both: initial pharmacotherapy for type 2 diabetes, a randomised controlled trial. Int $\mathcal{F}$ Clin Pract 66: 446-456.

International Diabetes Federation (2012) Global guideline for type 2 diabetes. Brussels: International Diabetes Federation. Available at: http://www.idf. org/new-global-guideline-type-2-diabetes-out-now-0 (Accessed 27 October 2014).

International Diabetes Federation (2013) IDF atlas, 6th edn. Brussels: International Diabetes Federation. Available at: http://www.idf.org/diabetesatlas (accessed 27 October 2014).

Inzucchi, S., Bergenstal, R., Buse, J., Diamant, M., Ferrannini, E., Nauck, M. et al. (2012) Management of hyperglycemia in type 2 diabetes: a patient-centered approach: position statement of the American Diabetes Association (ADA) and the European Association for the Study of Diabetes (EASD). Diabetes Care 35: 1364-1379.

Jabbour, S., Hardy, E., Sugg, J., Parikh, S. and Study 10 Group (2014) Dapagliflozin is effective as add-on therapy to sitagliptin with or without metformin: a 24-week, multicenter, randomized, double-blind, placebo-controlled study. Diabetes Care 37: 740-750.

Johnsson, K., Ptaszynska, A., Schmitz, B., Sugg, J., Parikh, S. and List, J. (2013a) Vulvovaginitis and balanitis in patients with diabetes treated with dapagliflozin. $\mathcal{F}$ Diabetes Complications 27: 479-484.

Johnsson, K., Ptaszynska, A., Schmitz, B., Sugg, J., Parikh, S. and List, J. (2013b) Urinary tract infections in patients with diabetes treated with dapagliflozin. f Diabetes Complications 27: 473-478.

Kasichayanula, S., Chang, M., Liu, X., Shyu, W., Griffen, S., Lacreta, F. et al. (2012) Lack of pharmacokinetic interactions between dapagliflozin and simvastatin, valsartan, warfarin, or digoxin. $A d v$ Ther 29: 163-177.

Kasichayanula, S., Liu, X., Shyu, W., Zhang, W., Pfister, M., Griffen, S. et al. (2011) Lack of pharmacokinetic interaction between dapagliflozin, a novel sodium-glucose transporter 2 inhibitor, and metformin, pioglitazone, glimepiride or sitagliptin in healthy subjects. Diabetes Obes Metab 13: 47-54. 
Kohan, D., Fioretto, P., Tang, W. and List, J. (2014) Long-term study of patients with type 2 diabetes and moderate renal impairment shows that dapagliflozin reduces weight and blood pressure but does not improve glycemic control. Kidney Int 85: 962-971.

Lambers Heerspink, H., De Zeeuw, D., Wie, L., Leslie, B. and List, J. (2013) Dapagliflozin a glucoseregulating drug with diuretic properties in subjects with type 2 diabetes. Diabetes Obes Metab 15: 853-862.

List, J. and Whaley, J. (2011) Glucose dynamics and mechanistic implications of SGLT2 inhibitors in animals and humans. Kidney Int 79: S20-27.

Matthaei, S., Rohwedder, K., Grohl, A. and Johnsson, E. (2013) Dapagliflozin improves glycaemic control and reduces body weight as add-on therapy to metformin plus sulphonylurea. Presented at European Association for the Study of Diabetes, Barcelona, Spain.

Nauck, M., Del Prato, S., Meier, J., Duran-Garcia, S., Rohwedder, K., Elze, M. et al. (2011) Dapagliflozin versus glipizide as add-on therapy in patients with type 2 diabetes who have inadequate glycemic control with metformin: a randomized, 52-week, double-blind, active-controlled noninferiority trial. Diabetes Care 34 2015-2022.

Nicolle, L., Capuano, G., Fung, A. and Usiskin, K. (2014) Urinary tract infection in randomized phase III studies of canagliflozin, a sodium glucose co-transporter 2 inhibitor. Postgrad Med 126: 7-17.

Nyirjesy, P., Zhao, Y., Ways, K. and Usiskin, K. (2012) Evaluation of vulvovaginal symptoms and Candida colonization in women with type 2 diabetes mellitus treated with canagliflozin, a sodium glucose co-transporter 2 inhibitor. Curr Med Res Opin 28: 1173-1178.

Ptaszynska, A., Johnsson, K., Parikh, S., De Bruin, T., Apanovitch, A. and List, J. (2014) Safety profile of dapagliflozin for type 2 diabetes: pooled analysis of clinical studies for overall safety and rare events. Drug Saf 37: 815-829.

Rosenstock, J., Vico, M., Wei, L., Salsali, A. and List, J. (2012) Effects of dapagliflozin, an SGLT2 inhibitor, on $\mathrm{HbA} 1 \mathrm{c}$, body weight, and hypoglycemia risk in patients with type 2 diabetes inadequately controlled on pioglitazone monotherapy. Diabetes Care 35: 1473-1478.

Rosenstock J., Hansen L., Zee P., Li Y., Cook W., Hirshberg B., Iqbal N. (2015) Dual add-on therapy in type 2 diabetes poorly controlled with metformin monotherapy: A randomized double-blind trial of saxagliptin plus dapagliflozin addition versus single addition of saxagliptin or dapagliflozin to metformin. Diabetes Care 38: 376-383.

Stark Casagrande, S., Fradkin, J., Saydah, S., Rust, K. and Cowie, C. (2013) The prevalence of meeting A1C, blood pressure, and LDL goals among people with diabetes, 1988-2010. Diabetes Care 36: 2271-2279.

Strojek, K., Yoon, K., Hruba, V., Elze, M., Langkilde, A. and Parikh, S. (2011) Effect of dapagliflozin in patients with type 2 diabetes who have inadequate glycaemic control with glimepiride: a randomized, 24-week, double-blind, placebo-controlled trial. Diabetes Obes Metab 13: 928-938.

Vasilakou, D., Karagiannis, T., Athanasiadou, E., Mainou, M., Liakos, A., Bekiari, E. et al. (2013) Sodium-glucose cotransporter 2 inhibitors for type 2 diabetes: a systematic review and meta-analysis. Ann Intern Med 159: 262-274.

Weber, M., Mansfield, T., Alessi, F. and Ptaszynska, A. (2013a) Effects of dapagliflozin on blood pressure in diabetic patients with hypertension inadequately controlled by a renin-angiotensin system blocker. Circulation 128: abstract A13144.

Weber, M., Mansfield, T., T'joen, C. and Ptaszynska, A. (2013b) Dapagliflozin for reduction of blood pressure in diabetic patients inadequately controlled with combination antihypertensive regimen. Circulation 128: abstract A13165.

Wilding, J., Woo, V., Rohwedder, K., Sugg, J. and Parikh, S. (2014) Dapagliflozin in patients with type 2 diabetes receiving high doses of insulin: efficacy and safety over two years. Diabetes Obes Metab 16: 124-136.

Wilding, J., Woo, V., Soler, N., Pahor, A., Sugg, J., Rohwedder, K. et al. (2012) Long-term efficacy of dapagliflozin in patients with type 2 diabetes mellitus receiving high doses of insulin. Ann Intern Med 156: 405-415.

Yale, J., Bakris, G., Cariou, B., Yue, D., DavidNeto, E., Xi, L. et al. (2013) Efficacy and safety of canagliflozin in subjects with type 2 diabetes and chronic kidney disease. Diabetes Obes Metab 15: 463-473.

Zhang, L., Feng, Y., List, J., Kasichayanula, S. and Pfister, M. (2010) Dapagliflozin treatment in patients with different stages of type 2 diabetes mellitus: effects on glycaemic control and body weight. Diabetes Obes Metab 12: 510-516. 